\title{
Chen's double sieve, Goldbach's conjecture and the twin prime problem, 2
}

by

\section{J. Wu (Nancy)}

1. Introduction. Let $\Omega(n)$ be the number of all prime factors of the integer $n$ with the convention $\Omega(1)=0$. For each even integer $N \geq 4$, we define

$$
D(N):=|\{p \leq N: \Omega(N-p)=1\}| ;
$$

here and in what follows, the letter $p$, with or without subscript, denotes a prime number. The well known Goldbach conjecture can be stated as $D(N) \geq 1$ for all even integers $N \geq 4$. A more precise version of this conjecture was proposed by Hardy \& Littlewood [10]:

$$
D(N) \sim 2 \Theta(N) \quad(N \rightarrow \infty),
$$

where

$$
\Theta(N):=\frac{C_{N} N}{(\log N)^{2}}, \quad C_{N}:=\prod_{p \mid N, p>2} \frac{p-1}{p-2} \prod_{p>2}\left(1-\frac{1}{(p-1)^{2}}\right) .
$$

Certainly, the asymptotic formula (1.1) is extremely difficult. One way of approaching the lower bound problem in (1.1) is to give a non-trivial lower bound for the quantity

$$
D_{1,2}(N):=|\{p \leq N: \Omega(N-p) \leq 2\}| .
$$

In this direction, Chen [5] proved, by his system of weights and the switching principle, the following famous theorem: Every sufficiently large even integer can be written as sum of a prime and an integer having at most two prime factors. More precisely, he established

$$
D_{1,2}(N) \geq 0.67 \Theta(N)
$$

2000 Mathematics Subject Classification: 11P32, 11N35, 11N05.

Key words and phrases: Chen's double sieve, Goldbach conjecture, twin prime problem. 
for $N \geq N_{0}$. As Halberstam \& Richert indicated in [9], it would be interesting to know whether a more elaborate weighting procedure could be adapted to the purpose of (1.3). This might lead to numerical improvements and could be important. Chen's constant 0.67 has been improved by many authors. The historical record is as follows:
0.689 by Halberstam \& Richert [9],
0.754 by Chen [6],
0.81 by Chen [7],
0.828 by Cai \& $\mathrm{Lu}[4]$,
0.836 by $\mathrm{Wu}[13]$,
0.867 by Cai $[2]$.

The aim of this paper is to propose a better constant.

TheOREM. For sufficiently large $N$, we have

$$
D_{1,2}(N) \geq 0.899 \Theta(N) .
$$

Our improvement comes from a delicate application of Chen's double sieve ([8], [12], [13]), which can be described as follows: With standard notation in the theory of sieve methods, the linear sieve formulas (see [9], or Lemma 2.2 of [13]) can be stated as

$$
X V(z) f\left(\frac{\log Q}{\log z}\right)+\text { error } \leq S(\mathcal{A} ; \mathcal{P}, z) \leq X V(z) F\left(\frac{\log Q}{\log z}\right)+\text { error. }
$$

These inequalities are the best possible in the sense that for

$$
\mathcal{A}=\mathcal{B}_{\nu}:=\{n \leq x: \Omega(n) \equiv \nu(\bmod 2)\} \quad(\nu=1,2),
$$

the upper and lower bounds in (1.4) are respectively attained by $\nu=1$ and $\nu=2$ (see [9, p. 239]). Aiming at improving Bombieri-Davenport's upper bound [1]

$$
D(N) \leq\{8+o(1)\} \Theta(N),
$$

Chen [8] found an improvement for (1.4) for some special sequences $\mathcal{A}$. Roughly speaking, for the sequence

$$
\mathcal{A}=\{N-p: p \leq N\}
$$

he narrowed down the gap in (1.4) by introducing two functions $h(s)$ and $H(s)$ such that the functions $s f(s) /\left(2 e^{\gamma}\right)$ and $s F(s) /\left(2 e^{\gamma}\right)$ are replaced by $s f(s) /\left(2 e^{\gamma}\right)+h(s)$ and $s F(s) /\left(2 e^{\gamma}\right)-H(s)$ respectively, where $\gamma$ is the Euler constant. The key point is thus to prove $h(s)>0$ and $H(s)>0$. Chen's proof is very long and difficult to follow, but his innovative idea is clear (see [11] for example). In [13], we gave a more comprehensive treatment of this method and named it Chen's double sieve. Indeed, our treatment is not 
only simpler but even more powerful than Chen's. Our approach improved Chen's upper estimate $D(N) \leq 7.8342 \Theta(N)$ to $D(N) \leq 7.8209 \Theta(N)$. It is worth indicating that Chen's record stood for 26 years before our work [13].

To prove our Theorem, we first simplify and improve Chen's weight system (cf. (12) of [7] and Lemma 2.2 below), and then apply Chen's double sieve, as the classical linear sieve, to handle terms such as $\Upsilon_{2}, \Upsilon_{3}, \Upsilon_{4}, \Upsilon_{5}$ and $\Upsilon_{6}$ in Propositions 4.1-4.4 below. The idea of using Chen's double sieve to treat sums of the type

$$
\sum_{\substack{N_{1}^{\phi_{1}} \leq p<N^{\phi_{2}} \\(p, N)=1}} S\left(\mathcal{A}_{p} ; \mathcal{P}(N), N^{\kappa}\right)
$$

first appeared in [12]. However, due to the first condition in (3.1) below, our Chen's double sieve can only handle the initial part of the sum over small $p$ in (1.5) (i.e. $p \leq N^{1 / 4}$ ). On the other hand, very recently Cai [2] used a similar idea to control the sum over large $p$ in (1.5). Actually his method can be viewed as a simplified version of Chen's double sieve (see Proposition 4.4 below and the comments before it). Here we shall combine both versions and refine them to obtain our result. As is apparent from the proof, the first version gives a saving of 0.0211 while the second saves 0.0078 . Without Chen's double sieve technique, we still obtain 0.870 in place of 0.899 , which is slightly better than Cai's 0.867 .

Clearly our method can be used to refine the corresponding constants in the conjugate problems ([2] and [3]). The proofs are very similar and even easier and simpler. Hence we omit the relevant discussion. Maybe this is a good exercise for senior graduate students in analytic number theory.

2. Chen's system of weights. This section is devoted to discussing the weighted sieve of Chen type. Let

$$
\mathcal{A}:=\{N-p: p \leq N\} \quad \text { and } \quad \mathcal{P}(N):=\{p:(p, N)=1\} .
$$

The sieve function is defined as

$$
S(\mathcal{A} ; \mathcal{P}(N), z):=|\{a \in \mathcal{A}:(a, P(z))=1\}|,
$$

where $P(z):=\prod_{p \leq z, p \in \mathcal{P}(N)} p$.

Lemma 2.1. Let $0<\kappa<\sigma \leq 1 / 3$. Then

$$
\begin{gathered}
2 D_{1,2}(N) \geq 2 S\left(\mathcal{A} ; \mathcal{P}(N), N^{\kappa}\right)-S_{1}(\kappa, \sigma)-2 S_{2}(\kappa, \sigma) \\
-S_{3}(\kappa, \sigma)+S_{4}(\kappa, \sigma)+O\left(N^{1-\kappa}\right),
\end{gathered}
$$


where

$$
\begin{aligned}
& S_{1}(\kappa, \sigma):=\sum_{\substack{N^{\kappa} \leq p<N^{\sigma} \\
(p, N)=1}} S\left(\mathcal{A}_{p} ; \mathcal{P}(N), N^{\kappa}\right), \\
& S_{2}(\kappa, \sigma):=\sum_{\substack{N^{\sigma} \leq p_{1}<p_{2}<\left(N / p_{1}\right)^{1 / 2} \\
\left(p_{1} p_{2}, N\right)=1}} S\left(\mathcal{A}_{p_{1} p_{2}} ; \mathcal{P}\left(N p_{1}\right), p_{2}\right), \\
& S_{3}(\kappa, \sigma):=\sum_{\substack{N^{\kappa} \leq p_{1}<N^{\sigma} \leq p_{2}<\left(N / p_{1}\right)^{1 / 2} \\
\left(p_{1} p_{2}, N\right)=1}} S\left(\mathcal{A}_{p_{1} p_{2}} ; \mathcal{P}\left(N p_{1}\right), p_{2}\right), \\
& S_{4}(\kappa, \sigma):=\sum_{\substack{N^{\kappa} \leq p_{1}<p_{2}<p_{3}<N^{\sigma} \\
\left(p_{1} p_{2} p_{3}, N\right)=1}} S\left(\mathcal{A}_{p_{1} p_{2} p_{3}} ; \mathcal{P}\left(N p_{1}\right), p_{2}\right) .
\end{aligned}
$$

The inequality (2.1) first appeared in [7, p. 479, (11)] with $(\kappa, \sigma)=$ $\left(\frac{1}{12}, \frac{1}{3.047}\right),\left(\frac{1}{9.2}, \frac{1}{3.41}\right)$ without proof. Cai \& [Lu] [4] gave a proof with an extra assumption $3 \sigma+\kappa>1$. In [13], we proved (2.1) under the hypothesis $0<\kappa<\sigma<1 / 3$. Clearly the proof there is also valid for $\sigma=1 / 3$. Very recently Cai [2] gave another proof for Lemma 2.1.

As in [7], we shall apply (2.1) with two different pairs of parameters $(\kappa, \sigma)$ to take advantage of $S_{4}(\kappa, \sigma)$. Our weighted sieve is simpler and more powerful than those of Chen $([7,(12)])$ and Cai $([2$, Lemma 6$])$.

Lemma 2.2. Let $\kappa_{2}>\kappa_{1} \geq 1 / 18$ be such that

$$
3 \kappa_{1}+\kappa_{2}<1 / 2 \text { and } 3 \kappa_{1}-\kappa_{2}<1 / 6 \text {. }
$$

Then

$$
\begin{aligned}
4 D_{1,2}(N) \geq & 3 \Upsilon_{1}+\Upsilon_{2}-\Upsilon_{3}-\Upsilon_{4}+\Upsilon_{5}+\Upsilon_{6} \\
& -2 \Upsilon_{7}-\Upsilon_{8}-\Upsilon_{9}-\Upsilon_{10}-\Upsilon_{11}+O\left(N^{1-\kappa_{1}}\right),
\end{aligned}
$$

where

$$
\begin{aligned}
& \Upsilon_{i}:=S\left(\mathcal{A} ; \mathcal{P}(N), N^{\kappa_{i}}\right) \quad(i=1,2), \\
& \Upsilon_{3}:=\sum_{\substack{N^{\kappa_{1}} \leq p<N^{1 / 3} \\
(p, N)=1}} S\left(\mathcal{A}_{p} ; \mathcal{P}(N), N^{\kappa_{1}}\right), \\
& \Upsilon_{4}:=\sum_{\substack{N^{\kappa_{1}} \leq p<N^{1 / 2-3 \kappa_{1}} \\
(p, N)=1}} S\left(\mathcal{A}_{p} ; \mathcal{P}(N), N^{\kappa_{1}}\right), \\
& \Upsilon_{5}:=\sum_{\substack{N_{1} \leq p_{1}<p_{2}<N^{\kappa_{2}} \\
\left(p_{1} p_{2}, N\right)=1}} S\left(\mathcal{A}_{p_{1} p_{2}} ; \mathcal{P}(N), N^{\kappa_{1}}\right),
\end{aligned}
$$




$$
\begin{aligned}
& \Upsilon_{6}:=\sum_{\substack{N^{\kappa_{1}} \leq p_{1}<N^{\kappa_{2}} \leq p_{2}<N^{1 / 2-3 \kappa_{1}} \\
\left(p_{1} p_{2}, N\right)=1}} S\left(\mathcal{A}_{p_{1} p_{2}} ; \mathcal{P}(N), N^{\kappa_{1}}\right), \\
& \Upsilon_{7}:=\sum_{\substack{N^{1 / 2-3 \kappa} 1 \leq p_{1}<p_{2}<\left(N / p_{1}\right)^{1 / 2} \\
\left(p_{1} p_{2}, N\right)=1}} S\left(\mathcal{A}_{p_{1} p_{2}} ; \mathcal{P}\left(N p_{1}\right), p_{2}\right), \\
& \Upsilon_{8}:=\sum_{\substack{N^{\kappa} \leq p_{1}<N^{1 / 3} \leq p_{2}<\left(N / p_{1}\right)^{1 / 2} \\
\left(p_{1} p_{2}, N\right)=1}} S\left(\mathcal{A}_{p_{1} p_{2}} ; \mathcal{P}\left(N p_{1}\right), p_{2}\right),
\end{aligned}
$$

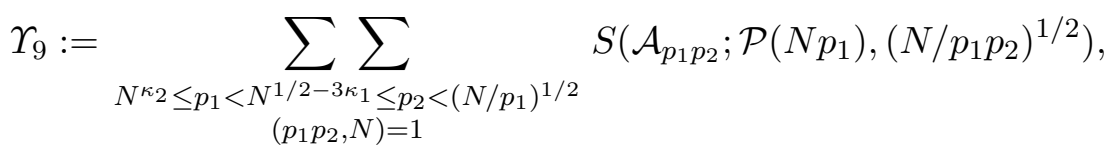

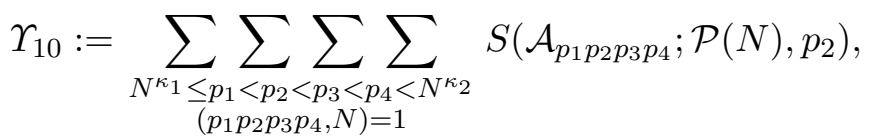

$$
\begin{aligned}
& \Upsilon_{11}:=\sum_{\substack{N^{\kappa_{1}} \leq p_{1}<p_{2}<p_{3}<N^{\kappa_{2}} \leq p_{4}<N^{1 / 2-2 \kappa_{1} / p_{3}} \\
\left(p_{1} p_{2} p_{3} p_{4}, N\right)=1}} S\left(\mathcal{A}_{p_{1} p_{2} p_{3} p_{4}} ; \mathcal{P}(N), p_{2}\right) .
\end{aligned}
$$

Proof. By noticing that our hypothesis implies $\kappa_{2}<1 / 2-3 \kappa_{1} \leq 1 / 3$, we can apply $(2.1)$ with $(\kappa, \sigma)=\left(\kappa_{2}, 1 / 2-3 \kappa_{1}\right)$ to obtain

$$
\begin{aligned}
2 D_{1,2}(N) \geq & 2 \Upsilon_{2}-S_{1}\left(\kappa_{2}, 1 / 2-3 \kappa_{1}\right) \\
& -2 \Upsilon_{7}-S_{3}\left(\kappa_{2}, 1 / 2-3 \kappa_{1}\right)+O\left(N^{1-\kappa_{2}}\right),
\end{aligned}
$$

where the term $S_{4}\left(\kappa_{2}, 1 / 2-3 \kappa_{1}\right)$ is dropped by non-negativity.

Buchstab's identity, applied three times, gives the equality

$$
\begin{aligned}
\Upsilon_{2}= & \Upsilon_{1}-\sum_{\substack{N^{\kappa_{1}} \leq p<N^{\kappa_{2}} \\
(p, N)=1}} S\left(\mathcal{A}_{p} ; \mathcal{P}(N), N^{\kappa_{1}}\right)+\Upsilon_{5} \\
& -\sum_{\substack{N_{1}{ }_{1} \leq p_{1}<p_{2}<p_{3}<N^{\kappa_{2}} \\
\left(p_{1} p_{2} p_{3}, N\right)=1}} \sum_{\left(\mathcal{A}_{p_{1} p_{2} p_{3}} ; \mathcal{P}(N), p_{1}\right) .} \sum,
\end{aligned}
$$

Similarly, a double application of Buchstab's identity yields

$$
\begin{aligned}
S_{1}\left(\kappa_{2}, 1 / 2-3 \kappa_{1}\right)= & \sum_{\substack{N^{\kappa_{2}} \leq p<N^{1 / 2-3 \kappa_{1}} \\
(p, N)=1}} S\left(\mathcal{A}_{p} ; \mathcal{P}(N), N^{\kappa_{1}}\right)-\Upsilon_{6} \\
& +\sum_{\substack{N^{\kappa_{1}} \leq p_{1}<p_{2}<N^{\kappa_{2}} \leq p_{3}<N^{1 / 2-3 \kappa_{1}} \\
\left(p_{1} p_{2} p_{3}, N\right)=1}} S\left(\mathcal{A}_{p_{1} p_{2} p_{3}} ; \mathcal{P}(N), p_{1}\right) .
\end{aligned}
$$


By Buchstab's identity, we can prove

$$
\begin{aligned}
& S_{3}\left(\kappa_{2}, 1 / 2-3 \kappa_{1}\right)
\end{aligned}
$$

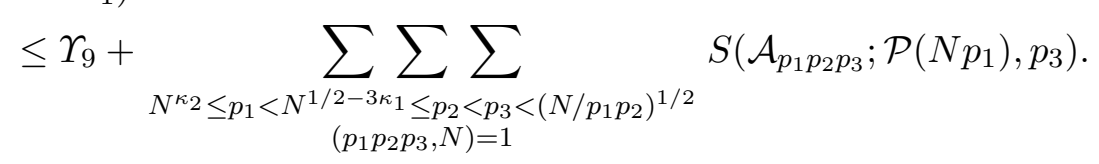

Inserting them into (2.3), we find that

$$
2 D_{1,2}(N) \geq \Upsilon_{1}+\Upsilon_{2}-\Upsilon_{4}+\Upsilon_{5}+\Upsilon_{6}-2 \Upsilon_{7}-\Upsilon_{9}-\Delta_{1}+O\left(N^{1-\kappa_{2}}\right)
$$

where

$$
\begin{aligned}
& \Delta_{1}:=\sum_{\substack{N_{1} \leq p_{1}<p_{2}<p_{3}<N^{\kappa_{2}} \\
\left(p_{1} p_{2} p_{3}, N\right)=1}} S\left(\mathcal{A}_{p_{1} p_{2} p_{3}} ; \mathcal{P}(N), p_{1}\right) \\
& +\sum_{\substack{N^{\kappa_{1}} \leq p_{1}<p_{2}<N^{\kappa_{2}} \leq p_{3}<N^{1 / 2-3 \kappa_{1}} \\
\left(p_{1} p_{2} p_{3}, N\right)=1}} S\left(\mathcal{A}_{p_{1} p_{2} p_{3}} ; \mathcal{P}(N), p_{1}\right)
\end{aligned}
$$

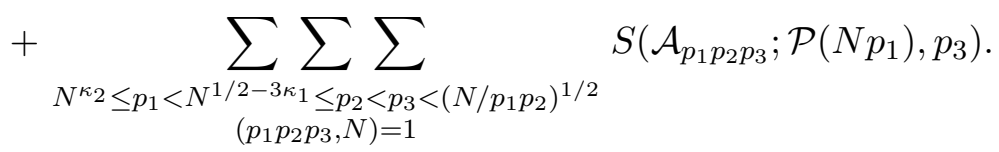

The inequality $(2.1)$ with $(\kappa, \sigma)=\left(\kappa_{1}, 1 / 3\right)$ gives

$$
2 D_{1,2}(N) \geq 2 \Upsilon_{1}-\Upsilon_{3}-\Upsilon_{8}+S_{4}\left(\kappa_{1}, 1 / 3\right)+O\left(N^{1-\kappa_{1}}\right),
$$

where we have used the fact that $S_{2}\left(\kappa_{1}, 1 / 3\right)=0$.

Adding (2.4) to (2.5) yields

$$
\begin{aligned}
4 D_{1,2}(N) \geq & 3 \Upsilon_{1}+\Upsilon_{2}-\Upsilon_{3}-\Upsilon_{4}+\Upsilon_{5}+\Upsilon_{6} \\
& -2 \Upsilon_{7}-\Upsilon_{8}-\Upsilon_{9}+\Delta_{2}+O\left(N^{1-\kappa_{1}}\right)
\end{aligned}
$$

where

$$
\Delta_{2}:=\sum_{\substack { N^{\kappa} \\
\begin{subarray}{c}{\leq p_{1}<p_{2}<p_{3}<N^{1 / 3} \\
\left(p_{1} p_{2} p_{3}, N\right)=1{ N ^ { \kappa } \\
\begin{subarray} { c } { \leq p _ { 1 } < p _ { 2 } < p _ { 3 } < N ^ { 1 / 3 } \\
( p _ { 1 } p _ { 2 } p _ { 3 } , N ) = 1 } }\end{subarray}} S\left(\mathcal{A}_{p_{1} p_{2} p_{3}} ; \mathcal{P}(N), p_{2}\right)-\Delta_{1} .
$$

Clearly all the summation ranges in the three triple sums of $\Delta_{1}$ are distinct and the first two are covered by the range of the triple sum in $\Delta_{2}$ (since our hypothesis on $\kappa_{1}$ and $\kappa_{2}$ implies $\max \left\{\kappa_{2}, 1 / 2-3 \kappa_{1}\right\} \leq 1 / 3$ ). On the other hand, we easily see that the range of summation in the third triple sum of $\Delta_{1}$ is equivalent to $N^{\kappa_{2}} \leq p_{1}<N^{1 / 2-3 \kappa_{1}} \leq p_{2} \leq\left(N / p_{1}\right)^{1 / 3}$ and $p_{2}<p_{3}<\left(N / p_{1} p_{2}\right)^{1 / 2}$. From this we deduce that $\left(N / p_{1} p_{2}\right)^{1 / 2} \leq$ $N^{\left(1 / 2+3 \kappa_{1}-\kappa_{2}\right) / 2} \leq N^{1 / 3}$, since $3 \kappa_{1}-\kappa_{2}<1 / 6$. Thus this range is also contained in the triple sum of $\Delta_{2}$. Therefore we have 


$$
\begin{aligned}
& \Delta_{2} \geq-\sum_{\substack{N^{\kappa} 1 \leq p_{1}<p_{2}<p_{3}<N^{\kappa_{2}} \\
\left(p_{1} p_{2} p_{3}, N\right)=1}} \Delta_{2}^{\prime} \\
& -\sum_{\substack{N^{\kappa_{1}} \leq p_{1}<p_{2}<N^{\kappa_{2}} \leq p_{3}<N^{1 / 2-2 \kappa_{1} / p_{2}} \\
\left(p_{1} p_{2} p_{3}, N\right)=1}} \Delta_{2}^{\prime}
\end{aligned}
$$

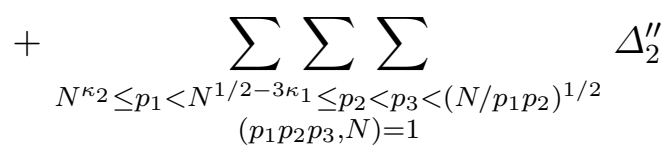

$$
\begin{aligned}
& \geq-\Upsilon_{10}-\Upsilon_{11}+O\left(N^{1-\kappa_{1}}\right)
\end{aligned}
$$

where

$$
\begin{aligned}
& \Delta_{2}^{\prime}:=S\left(\mathcal{A}_{p_{1} p_{2} p_{3}} ; \mathcal{P}(N), p_{1}\right)-S\left(\mathcal{A}_{p_{1} p_{2} p_{3}} ; \mathcal{P}(N), p_{2}\right), \\
& \Delta_{2}^{\prime \prime}:=S\left(\mathcal{A}_{p_{1} p_{2} p_{3}} ; \mathcal{P}(N), p_{2}\right)-S\left(\mathcal{A}_{p_{1} p_{2} p_{3}} ; \mathcal{P}(N), p_{3}\right)
\end{aligned}
$$

Combining this with (2.6), we obtain the required result.

REMARK 1. In the proof, we have chosen $(\kappa, \sigma)=\left(\kappa_{1}, 1 / 2-3 \kappa_{1}\right)$, $\left(\kappa_{2}, 1 / 3\right)$ when applying Lemma 2.1 . It is possible to optimize the choice of $\sigma$. But this increases the number of terms of (2.2), and the numerical improvement for the Theorem is quite small.

3. Chen's double sieve. In this section, we recall Chen's double sieve described in [13] and give numerical lower bounds for $H(s)$ and $h(s)$ for later use.

For any large even integer $N$, we write

$$
\mathcal{A}:=\{N-p: p \leq N\}, \quad \mathcal{P}(N):=\{p:(p, N)=1\} .
$$

Let $\delta>0$ be a sufficiently small number $\left(^{1}\right)$ and $k \in \mathbb{Z}^{+}$. Put

$$
Q:=N^{1 / 2-\delta}, \quad \underline{d}:=Q / d, \quad \mathcal{L}:=\log N, \quad W_{k}:=N^{\delta^{1+k}} .
$$

Denote by $\pi_{[Y, Z)}$ the characteristic function of the set $\mathcal{P}(N) \cap[Y, Z)$. For $k \in \mathbb{Z}^{+}$and $N \geq 2$, let $\mathfrak{U}_{k}(N)$ be the set of all arithmetical functions $\sigma$ which can be written in the form

$$
\sigma=\pi_{\left[V_{1} / \Delta, V_{1}\right)} * \cdots * \pi_{\left[V_{i} / \Delta, V_{i}\right)}
$$

where $\Delta$ is a real number with $1+\mathcal{L}^{-4} \leq \Delta<1+2 \mathcal{L}^{-4}, i$ is an integer with $0 \leq i \leq k$, and $V_{1}, \ldots, V_{i}$ are real numbers satisfying

$\left({ }^{1}\right)$ In numerical computation, we can formally take $\delta=0$. 


$$
\left\{\begin{array}{l}
V_{1}^{2} \leq Q \\
V_{1} V_{2}^{2} \leq Q \\
\cdots \cdots \cdots \cdots \\
V_{1} \cdots V_{i-1} V_{i}^{2} \leq Q, \\
V_{1} \geq \cdots \geq V_{i} \geq W_{k} .
\end{array}\right.
$$

We adopt the convention that $\sigma$ is the characteristic function of the set $\{1\}$ if $i=0$.

Let $F$ and $f$ be defined by

$$
\begin{array}{cll}
F(s)=2 e^{\gamma} / s, & f(s)=0 & (0<s \leq 2), \\
(s F(s))^{\prime}=f(s-1), & (s f(s))^{\prime}=F(s-1) & (s>2),
\end{array}
$$

where $\gamma$ is Euler's constant. Moreover, we set

$$
A(s):=s F(s) /\left(2 e^{\gamma}\right), \quad a(s):=s f(s) /\left(2 e^{\gamma}\right),
$$

and introduce the notation

$$
\begin{gathered}
\Phi(N, \sigma, s):=\sum_{d} \sigma(d) S\left(\mathcal{A}_{d} ; \mathcal{P}(d N), \underline{d}^{1 / s}\right), \\
\Theta(N, \sigma):=4 \operatorname{li}(N) \sum_{d} \frac{\sigma(d) C_{d N}}{\varphi(d) \log \underline{d}},
\end{gathered}
$$

where $\varphi(d)$ is the Euler function.

For $k \in \mathbb{Z}^{+}, N_{0} \geq 2$ and $s \in[1,10]$, we define $H_{k, N_{0}}(s)$ and $h_{k, N_{0}}(s)$ to be the supremum of $h \geq-\infty$ such that for all $N \geq N_{0}$ and $\sigma \in \mathfrak{U}_{k}(N)$, the inequalities

$$
\begin{aligned}
& \Phi(N, \sigma, s) \leq\{A(s)-h\} \Theta(N, \sigma), \\
& \Phi(N, \sigma, s) \geq\{a(s)+h\} \Theta(N, \sigma)
\end{aligned}
$$

hold true respectively. Obviously $H_{k, N_{0}}(s)$ and $h_{k, N_{0}}(s)$ are decreasing in $N_{0}$, as well as decreasing in $k$ by Lemma 3.1. Hence their limits at infinity exist (in the extended real line), and we write

$$
\begin{aligned}
H_{k}(s) & :=\lim _{N_{0} \rightarrow \infty} H_{k, N_{0}}(s), & H(s) & :=\lim _{k \rightarrow \infty} H_{k}(s), \\
h_{k}(s) & :=\lim _{N_{0} \rightarrow \infty} h_{k, N_{0}}(s), & h(s) & :=\lim _{k \rightarrow \infty} h_{k}(s) .
\end{aligned}
$$

The next lemma collects the relevant properties of these functions (see [13, Lemma 3.2, Propositions $1 \& 2$ and Corollary 1]).

LEMMA 3.1.

(i) For $k \in \mathbb{Z}^{+}, N \geq N_{0}, s \in[1,10]$ and $\sigma \in \mathfrak{U}_{k}(N)$, we have

$$
\begin{aligned}
& \Phi(N, \sigma, s) \leq\left\{A(s)-H_{k, N_{0}}(s)\right\} \Theta(N, \sigma), \\
& \Phi(N, \sigma, s) \geq\left\{a(s)+h_{k, N_{0}}(s)\right\} \Theta(N, \sigma) .
\end{aligned}
$$


(ii) For $k \in \mathbb{Z}^{+}$and $s \in[1,10]$, we have $H_{k}(s) \geq 0$ and $h_{k}(s) \geq 0$.

(iii) For $2 \leq s \leq s^{\prime} \leq 10$, we have

$$
\begin{aligned}
& h(s) \geq h\left(s^{\prime}\right)+\int_{s-1}^{s^{\prime}-1} \frac{H(t)}{t} d t, \\
& H(s) \geq H\left(s^{\prime}\right)+\int_{s-1}^{s^{\prime}-1} \frac{h(t)}{t} d t .
\end{aligned}
$$

(iv) The function $H(s)$ is decreasing on $[1,10]$. The function $h(s)$ is increasing on $[1,2]$ and decreasing on $[2,10]$.

We cannot give explicit expressions for $H(s)$ and $h(s)$. But it is possible to obtain numerical lower bounds for these two functions. Let

$$
s_{i}:=2+0.1 i \quad(i \geq 0) .
$$

By $\left[13, \S 7\right.$, we have numerical lower bounds of $H\left(s_{i}\right)$ for $2 \leq i \leq 10$. Next we shall consider the case of $11 \leq i \leq 29$ and the lower bounds of $h\left(s_{i}\right)$ for $0 \leq i \leq 29$. These will be used in the proof of the Theorem.

Let $\mathbf{1}_{[a, b]}(t)$ be the characteristic function of the interval $[a, b]$. Define

$$
\sigma(a, b, c):=\int_{a}^{b} \log \left(\frac{c}{t-1}\right) \frac{d t}{t}, \quad \sigma_{0}(t):=\frac{\sigma(3, t+2, t+1)}{1-\sigma(3,5,4)} .
$$

From (6.2) of [13] and the decreasing of $H(s)$, we deduce that

$$
H\left(s_{j}\right) \geq \sum_{2 \leq i \leq 10} c_{i, j} H\left(s_{i}\right)
$$

for $11 \leq j \leq 29$, where

$$
c_{2, j}:=\int_{1}^{s_{2}}\left\{\frac{\sigma_{0}(t)}{t} \log \left(\frac{4}{s_{j}-1}\right)+\frac{\mathbf{1}_{\left[s_{j}-2,3\right]}(t)}{t} \log \left(\frac{t+1}{s_{j}-1}\right)\right\} d t
$$

and

$$
c_{i, j}:=\int_{s_{i-1}}^{s_{i}}\left\{\frac{\sigma_{0}(t)}{t} \log \left(\frac{4}{s_{j}-1}\right)+\frac{\mathbf{1}_{\left[s_{j}-2,3\right]}(t)}{t} \log \left(\frac{t+1}{s_{j}-1}\right)\right\} d t
$$

for $3 \leq i \leq 10$. From (3.8) and the fact that $h(s) \geq 0$, we also derive

$$
\begin{aligned}
h\left(s_{j}\right) \geq \int_{s_{j}-1}^{5} \frac{H(t)}{t} d t \geq & H\left(s_{2}\right) \log \left(\frac{s_{\max \{2, j-10\}}}{s_{j}-1}\right) \\
& +\sum_{\max \{3, j-9\} \leq i \leq 29} H\left(s_{i}\right) \log \left(\frac{s_{i}}{s_{i-1}}\right)
\end{aligned}
$$

for $0 \leq j \leq 29$. 
Using the numerical lower bounds of $H\left(s_{i}\right)$ for $2 \leq i \leq 10$ given in [13, $\S 7],(3.11)$ and (3.12), we get via a numerical computation the following results.

Table 1. Numerical lower bounds for $H\left(s_{i}\right)$

\begin{tabular}{|c|c|c|c|c|c|c|c|c|}
\hline$i$ & $s_{i}$ & $H\left(s_{i}\right) \geq$ & $i$ & $s_{i}$ & $H\left(s_{i}\right) \geq$ & $i$ & $s_{i}$ & $H\left(s_{i}\right) \geq$ \\
\hline & & & 10 & 3.0 & 0.0072943 & 20 & 4.0 & 0.0010835 \\
\hline & & & 11 & 3.1 & 642 & 21 & 4.1 & 8451 \\
\hline 2 & 2.2 & 0.0223939 & 12 & 3.2 & 0.0052233 & 22 & 4.2 & 0.0006482 \\
\hline 3 & 2.3 & 0.0217196 & 13 & 3.3 & 0.0044073 & 23 & 4.3 & 0.0004882 \\
\hline 4 & 2.4 & 0.0202876 & 14 & 3.4 & 0.0036995 & 24 & 4.4 & 0.0003602 \\
\hline 5 & 2.5 & 0.0181433 & 15 & 3.5 & 0.0030860 & 25 & 4.5 & 0.0002592 \\
\hline 6 & 2.6 & 0.0158644 & 16 & 3.6 & 0.0025551 & 26 & 4.6 & 0.0001803 \\
\hline 7 & 2.7 & 0.0129923 & 17 & 3.7 & 0.0020972 & 27 & 4.7 & 0.0001187 \\
\hline 8 & 2.8 & 0.0100686 & 18 & 3.8 & 0.0017038 & 28 & 4.8 & 0.0000702 \\
\hline 9 & 2.9 & 0.0078162 & 19 & 3.9 & 0.0013680 & 29 & 4.9 & 0.0000313 \\
\hline
\end{tabular}

Table 2. Numerical lower bounds for $h\left(s_{i}\right)$

\begin{tabular}{|c|c|c|c|c|c|c|c|c|}
\hline$i$ & $s_{i}$ & $h\left(s_{i}\right) \geq$ & $i$ & $s_{i}$ & $h\left(s_{i}\right) \geq$ & $i$ & $s_{i}$ & $h\left(s_{i}\right) \geq$ \\
\hline 0 & 2.0 & 0.0232385 & 10 & 3.0 & 0.0077162 & 20 & 4.0 & 0.0010120 \\
\hline 1 & 2.1 & 0.0211041 & 11 & 3.1 & 0.0066236 & 21 & 4.1 & 0.0008099 \\
\hline 2 & 2.2 & 0.0191556 & 12 & 3.2 & 0.0055818 & 22 & 4.2 & 0.0006440 \\
\hline 3 & 2.3 & 0.0173631 & 13 & 3.3 & 0.0046164 & 23 & 4.3 & 0.0005084 \\
\hline 4 & 2.4 & 0.0157035 & 14 & 3. & 0.0037529 & 24 & 4.4 & 0.0003980 \\
\hline 5 & 2.5 & 0.0141585 & 15 & 3.5 & 0.0030123 & 25 & 4.5 & 0.0003085 \\
\hline 6 & 2.6 & 0.0127132 & 16 & 3.6 & 0.0023901 & 26 & 4.6 & 0.0002365 \\
\hline 7 & 2.7 & $0.011:$ & 17 & $3 .^{\prime}$ & 0.0018997 & 27 & 4.7 & 0.0001791 \\
\hline 8 & 2.8 & 0.0100756 & 18 & 3.8 & 0.0015336 & 28 & 4.8 & 0.0001336 \\
\hline 9 & 2.9 & 0.0088648 & 19 & 3.9 & 0.0012593 & 29 & 4.9 & 0.0000981 \\
\hline
\end{tabular}

REMARK 2. It is possible to get better numerical lower bounds for $H\left(s_{i}\right)$ and $h\left(s_{i}\right)$ by applying (3.8) and (3.9) repeatedly. But the improvement will be small.

4. Application of Chen's double sieve. In this section, we apply Chen's double sieve to estimate the terms $\Upsilon_{3}, \Upsilon_{4}, \Upsilon_{5}$ and $\Upsilon_{6}$ in (2.2). Propositions 4.1-4.4 below concern a general context. These estimates are better than those obtained by the classical linear sieve, since $H(s), h(s)>0$.

Proposition 4.1. Let $0<\phi_{1}<\phi_{2}<1 / 4$ and $\kappa>0$ be such that

$$
\phi_{2}+\kappa \leq 1 / 2 \text {. }
$$


Then for $N \rightarrow \infty$, we have

$$
\sum_{\substack{N_{1} \leq p<N^{\phi_{2}} \\(p, N)=1}} S\left(\mathcal{A}_{p} ; \mathcal{P}(N), N^{\kappa}\right) \leq\left\{8 \int_{\left(1 / 2-\phi_{2}\right) / \kappa}^{\left(1 / 2-\phi_{1}\right) / \kappa} \frac{A(t)-H(t)}{t(1-2 \kappa t)} d t+o(1)\right\} \Theta(N) .
$$

Proof. We keep the previous notation. Denote by $S$ the sum in the proposition. Let $\alpha_{j}:=N^{\phi_{1}} \Delta^{j}$ and $J$ be the integer such that $\alpha_{J} \leq N^{\phi_{2}}<\alpha_{J+1}$. We write

$$
S=\sum_{1 \leq j \leq J} \sum_{p} \pi_{\left[\alpha_{j-1}, \alpha_{j}\right)}(p) S\left(\mathcal{A}_{p} ; \mathcal{P}(p N), \underline{p}^{1 / \tau_{p}}\right)+R_{1},
$$

where $\tau_{p}:=(\log \underline{p}) /(\kappa \log N)$ and

$$
R_{1}:=\sum_{\alpha_{J} \leq p<N^{\phi_{2}}} S\left(\mathcal{A}_{p} ; \mathcal{P}(N), N^{\kappa}\right) \ll \sum_{\alpha_{J} \leq p<N^{\phi_{2}}} N / p \ll \Theta(N) \mathcal{L}^{-3} .
$$

Introducing

$$
\tau_{j}:=\left(\log \underline{\alpha_{j}}\right) /(\kappa \log N),
$$

we easily see that $\pi_{\left[\alpha_{j-1}, \alpha_{j}\right)}(p) \neq 0 \Rightarrow \tau_{j} \leq \tau_{p} \leq \tau_{j-1}$. Thus we can deduce from (4.1) and (4.2) that

$$
S \leq \sum_{1 \leq j \leq J} \sum_{p} \pi_{\left[\alpha_{j-1}, \alpha_{j}\right)}(p) S\left(\mathcal{A}_{p} ; \mathcal{P}(p N), \underline{p}^{1 / \tau_{j}}\right)+O\left(\Theta(N) \mathcal{L}^{-3}\right),
$$

where we have used the following estimates:

$$
\begin{aligned}
\sum_{1 \leq j \leq J} \sum_{p} \pi_{\left[\alpha_{j-1}, \alpha_{j}\right)}(p)\{S & \left.\left(\mathcal{A}_{p} ; \mathcal{P}(p N), \underline{p}^{1 / \tau_{p}}\right)-S\left(\mathcal{A}_{p} ; \mathcal{P}(p N), \underline{p}^{1 / \tau_{j}}\right)\right\} \\
& \leq \sum_{1 \leq j \leq J} \sum_{\alpha_{j-1} \leq p<\alpha_{j}} \sum_{\underline{p}^{1 / \tau_{p}} \leq p^{\prime}<\underline{p}^{1 / \tau_{j}}} N /\left(p p^{\prime}\right) \\
& \ll N \mathcal{L}^{-5} \sum_{1 \leq j \leq J} \sum_{\alpha_{j-1} \leq p<\alpha_{j}} 1 / p \ll \Theta(N) \mathcal{L}^{-3} .
\end{aligned}
$$

Next we treat the inner sum (over $p$ ) in (4.3). Clearly for each $j \in$ $\{1, \ldots, J\}$, our hypothesis on $\phi_{1}, \phi_{2}$ and $\kappa$ ensures that $\pi_{\left[\alpha_{j-1}, \alpha_{j}\right)} \in \mathfrak{U}_{k}(N)$ for all $k \geq 0, N_{0} \geq 2$ and $N \geq N_{0}$, and $\tau_{j} \geq 1$. Thus we can apply (3.6) of Lemma 3.1 to estimate the sum over $p$ (which is $\left.\Phi\left(N, \pi_{\left[\alpha_{j-1}, \alpha_{j}\right.}\right), \tau_{j}\right)$ ):

$$
\begin{aligned}
S & \leq \sum_{1 \leq j \leq J}\left\{A\left(\tau_{j}\right)-H_{k, N_{0}}\left(\tau_{j}\right)\right\} \Theta\left(N, \pi_{\left[\alpha_{j-1}, \alpha_{j}\right)}\right)+O\left(\Theta(N) \mathcal{L}^{-3}\right) \\
& \leq 4 \operatorname{li}(N) \frac{C_{N}}{\log \underline{\underline{1}}} \sum_{\alpha_{0} \leq p<\alpha_{J}} \frac{A\left(\tau_{p}\right)-H_{k, N_{0}}\left(\tau_{p}\right)}{(p-2)(1-\log p / \log \underline{1})}+O\left(\Theta(N) \mathcal{L}^{-3}\right) \\
& \leq 4 \operatorname{li}(N) \frac{C_{N}}{\log \underline{1}} \sum_{N^{\phi_{1}} \leq p<N^{\phi_{2}}} \frac{A\left(\tau_{p}\right)-H_{k, N_{0}}\left(\tau_{p}\right)}{(p-2)(1-\log p / \log \underline{1})}+O\left(\Theta(N) \mathcal{L}^{-3}\right),
\end{aligned}
$$


where we have used the fact that $A(s)-H_{k, N_{0}}(s)$ is increasing in $s$. An integration by parts with the prime number theorem shows that

$$
\sum_{N^{\phi_{1}} \leq p<N^{\phi_{2}}} \frac{A\left(\tau_{p}\right)-H_{k, N_{0}}\left(\tau_{p}\right)}{(p-2)(1-\log p / \log \underline{1})}=\int_{\left(1 / 2-\phi_{2}\right) / \kappa}^{\left(1 / 2-\phi_{1}\right) / \kappa} \frac{A(t)-H_{k, N_{0}}(t)}{t(1-2 \kappa t)} d t+O_{\delta, k}(\varepsilon) .
$$

Hence

$$
S \leq 8\left\{\int_{\left(1 / 2-\phi_{2}\right) / \kappa}^{\left(1 / 2-\phi_{1}\right) / \kappa} \frac{A(t)-H_{k, N_{0}}(t)}{t(1-2 \kappa t)} d t+O_{\delta, k}(\varepsilon)\right\} \Theta(N)
$$

for $N \geq N_{0}$. From this, we infer that

$$
\limsup _{N \rightarrow \infty} \frac{S}{\Theta(N)} \leq 8 \int_{\left(1 / 2-\phi_{2}\right) / \kappa}^{\left(1 / 2-\phi_{1}\right) / \kappa} \frac{A(t)-H_{k, N_{0}}(t)}{t(1-2 \kappa t)} d t+O_{\delta, k}(\varepsilon)
$$

which implies, by taking $N \rightarrow \infty, k \rightarrow \infty$ and $\varepsilon \rightarrow 0$,

$$
\limsup _{N \rightarrow \infty} \frac{S}{\Theta(N)} \leq 8 \int_{\left(1 / 2-\phi_{2}\right) / \kappa}^{\left(1 / 2-\phi_{1}\right) / \kappa} \frac{A(t)-H(t)}{t(1-2 \kappa t)} d t
$$

Clearly this is equivalent to the required inequality.

In a similar fashion we can prove the following results.

Proposition 4.2. Let $0<\phi_{1}<\phi_{2}<1 / 6$ and $\kappa>0$ be such that

$$
2 \phi_{2}+\kappa \leq 1 / 2 \text {. }
$$

Then for $N \rightarrow \infty$, we have

$$
\begin{aligned}
& \sum_{\substack{N^{\phi_{1}} \leq p_{1}<p_{2}<N^{\phi_{2}} \\
\left(p_{1} p_{2}, N\right)=1}} S\left(\mathcal{A}_{p_{1} p_{2}} ; \mathcal{P}(N), N^{\kappa}\right) \\
& \geq\left\{8 \int_{\phi_{1}\left(1 / 2-\phi_{2}-t\right) / \kappa}^{\phi_{2}} \int^{(1 / 2-2 t) / \kappa} \frac{a(u)+h(u)}{t(1-2 t-2 \kappa u)} d t d u+o(1)\right\} \Theta(N) .
\end{aligned}
$$

Proposition 4.3. Let $0<\phi_{1}<\phi_{2} \leq \phi_{3}<\phi_{4}<1 / 4$ and $\kappa>0$ be such that

$$
2 \phi_{2}+\phi_{4}<1 / 2 \text { and } \phi_{2}+\phi_{4}+\kappa \leq 1 / 2 \text {. }
$$

Then for $N \rightarrow \infty$, we have

$$
\begin{aligned}
\sum_{\substack{N^{\phi_{1}} \leq p_{1}<N^{\phi_{2}} \\
\left(p_{1} p_{2}, N\right)=1}} \sum_{N^{\phi_{3}} \leq p_{1}<N^{\phi_{4}}} S\left(\mathcal{A}_{p_{1} p_{2}} ; \mathcal{P}(N), N^{\kappa}\right) \\
\geq\left\{8 \int_{\phi_{1}\left(1 / 2-\phi_{4}-t\right) / \kappa}^{\phi_{2}\left(1 / 2-\phi_{3}-t\right) / \kappa} \frac{a(u)+h(u)}{t u(1-2 t-2 \kappa u)} d t d u+o(1)\right\} \Theta(N) .
\end{aligned}
$$


Finally, we estimate the sum (1.5) with $\phi_{1} \geq 1 / 4$. In this case, we cannot directly apply our delicate Chen's double sieve because of the first condition of (3.1). As remarked by Cai [2], it is possible to use a simplified version of Chen's double sieve. This approach will give a result better than using the classical linear sieve but weaker than Proposition 4.1, since, without iteration, $\Psi_{1}(s)$ or $\Psi_{2}(s)$ are principal contributions of $H(s)$. (See Lemmas 5.1 and 5.2 of [13] and compare Proposition 4.4 below and Proposition 4.1.)

Proposition 4.4. Let $\kappa>0, \phi>0$ and $2 \leq s \leq 3 \leq s^{\prime} \leq 5$ be such that

$$
1 / 4 \leq 1 / 2-s \kappa<\phi
$$

Then for $N \rightarrow \infty$, we have

$$
\begin{aligned}
& \sum_{\substack{N^{1 / 2-s \kappa} \leq p<N^{\phi} \\
(p, N)=1}} S\left(\mathcal{A}_{p} ; \mathcal{P}(N), N^{\kappa}\right) \\
& \leq\left\{8 \int_{(1 / 2-\phi) / \kappa}^{s} \frac{A(t)-\Psi_{1}(s)}{t(1-2 \kappa t)} d t+o(1)\right\} \Theta(N),
\end{aligned}
$$

where

$$
\begin{aligned}
\Psi_{1}(s):= & -\int_{2}^{s^{\prime}-1} \frac{\log (t-1)}{t} d t+\frac{1}{2} \int_{1-1 / s}^{1-1 / s^{\prime}} \frac{\log \left(s^{\prime} t-1\right)}{t(1-t)} d t \\
& -\max _{\phi \geq 2} \iiint_{1 / s^{\prime} \leq t \leq u \leq v \leq 1 / s} \omega\left(\frac{\phi-t-u-v}{u}\right) \frac{d t d u d v}{t u^{2} v}
\end{aligned}
$$

and $\omega(u)$ is Buchstab's function. The same result holds if we replace $\Psi_{1}(s)$ by $\Psi_{2}(s)$, where $\Psi_{2}(s)$ is defined as in Lemma 5.2 of [13].

Proof. For simplicity, we denote the sum by $S$. Since $N^{\kappa} \geq \underline{p}^{1 / s}$ for $p \geq N^{1 / 2-s \kappa}$, we can write

$$
\begin{aligned}
S & \leq \sum_{\substack{N^{1 / 2}-s \kappa \leq p<N^{\phi} \\
(p, N)=1}} S\left(\mathcal{A}_{p} ; \mathcal{P}(N), \underline{p}^{1 / s}\right) \\
& \leq \sum_{1 \leq j \leq J} \sum_{p} \pi_{\left[\alpha_{j-1}, \alpha_{j}\right)}(p) S\left(\mathcal{A}_{p} ; \mathcal{P}(N), \underline{p}^{1 / s}\right),
\end{aligned}
$$

where $\alpha_{j}:=N^{1 / 2-s \kappa} \Delta^{j}$ and $J$ is the integer such that $\alpha_{J-1} \leq N^{\phi}<\alpha_{J}$.

Similar to Lemma 4.1 of [13], we can prove that there is a constant $\eta>0$ such that

$$
S \leq \sum_{1 \leq j \leq J} \sum_{p} \pi_{\left[\alpha_{j-1}, \alpha_{j}\right)}(p)\left(\Omega_{1}(p)-\frac{1}{2} \Omega_{2}(p)+\frac{1}{2} \Omega_{3}(p)\right)+O\left(N^{1-\eta}\right),
$$


where

$$
\begin{aligned}
& \Omega_{1}(p):=S\left(\mathcal{A}_{p} ; \mathcal{P}(p N), \underline{p}^{1 / s^{\prime}}\right), \\
& \Omega_{2}(p):=\sum_{\substack{\underline{p}^{1 / s^{\prime}} \leq p_{1}<\underline{p}^{1 / s} \\
\left(p_{1}, N\right)=1}} S\left(\mathcal{A}_{p p_{1}} ; \mathcal{P}(p N), \underline{p}^{1 / s^{\prime}}\right),
\end{aligned}
$$

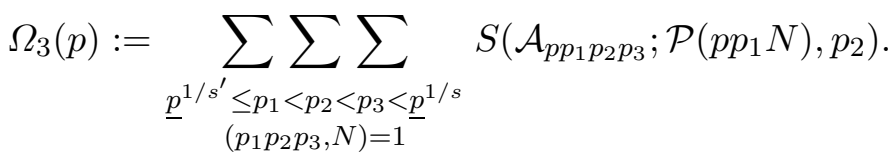

Similar to (5.1), (5.2) and (5.9) of [13], we can prove, uniformly for $N \geq 10$ and for $1 \leq i \leq 3,1 \leq j \leq J$,

$$
\sum_{p} \pi_{\left[\alpha_{j-1}, \alpha_{j}\right)}(p) \Omega_{i}(p) \leq\left\{\widetilde{\Omega}_{i}\left(s, s^{\prime}\right)+o(1)\right\} \Theta\left(N, \pi_{\left[\alpha_{j-1}, \alpha_{j}\right)}\right),
$$

where

$$
\begin{aligned}
& \widetilde{\Omega}_{1}\left(s, s^{\prime}\right):=A\left(s^{\prime}\right), \\
& \widetilde{\Omega}_{2}\left(s, s^{\prime}\right):=\int_{1-1 / s}^{1-1 / s^{\prime}} \frac{a\left(s^{\prime} t\right)}{t(1-t)} d t \\
& \widetilde{\Omega}_{3}\left(s, s^{\prime}\right):=2 \max _{\phi \geq 2} \iiint_{1 / s^{\prime} \leq t \leq u \leq v \leq 1 / s} \omega\left(\frac{\phi-t-u-v}{u}\right) \frac{d t d u d v}{t u^{2} v} .
\end{aligned}
$$

Inserting these into (4.4) and noticing that

$$
A\left(s^{\prime}\right)=1+\int_{2}^{s^{\prime}-1} \frac{\log (t-1)}{t} d t, \quad a\left(s^{\prime} t\right)=\log \left(s^{\prime} t-1\right),
$$

we find that

$$
\begin{aligned}
S & \leq\left\{1-\Psi_{1}(s)+o(1)\right\} \sum_{1 \leq j \leq J} \Theta\left(N, \pi_{\left[\alpha_{j-1}, \alpha_{j}\right)}\right)+O\left(N^{1-\eta}\right) \\
& \leq\left\{8\left(1-\Psi_{1}(s)\right) \int_{1 / 2-s \kappa}^{\phi} \frac{d t}{t(1-2 t)}+o(1)\right\} \Theta(N)
\end{aligned}
$$

which is equivalent to the required result for the case of $\Psi_{1}(s)$, since

$$
\int_{(1 / 2-\phi) / \kappa}^{s} \frac{A(t)}{t(1-2 \kappa t)} d t=\int_{(1 / 2-\phi) / \kappa}^{s} \frac{d t}{t(1-2 \kappa t)}=\int_{1 / 2-s \kappa}^{\phi} \frac{d t}{t(1-2 t)} .
$$

The case of $\Psi_{2}(s)$ can be treated in the same way. The main difference is the use of Lemma 4.2 of [13] in place of Lemma 4.1 there. We omit the details. 


\section{Proof of the Theorem. Set}

$$
\kappa_{1}=1 / 13.27 \text { and } \kappa_{2}=1 / 8.24,
$$

which satisfy the hypothesis of Lemma 2.2. Next we estimate all the terms $\Upsilon_{i}$ in $(2.2)$.

$1^{\circ}$ Lower bounds of $\Upsilon_{1}$ and $\Upsilon_{2}$. Write $N^{\kappa}=\underline{1}^{\kappa^{\prime}}$ with $\kappa^{\prime}:=\kappa /(1 / 2-\delta)$. By using (4.2) with $\sigma:=\mathbf{1}_{\{1\}}$ (the characteristic function of $\{1\}$ ), it follows that

$$
\begin{aligned}
\Upsilon_{i} & =\Phi\left(N, \mathbf{1}_{\{1\}}, 1 / \kappa_{i}^{\prime}\right) \geq\left\{a\left(1 / \kappa_{i}^{\prime}\right)+h_{k, N_{0}}\left(1 / \kappa_{i}^{\prime}\right)\right\} \Theta\left(N, \mathbf{1}_{\{1\}}\right) \\
& \geq\left\{F_{i}+o(1)\right\} \Theta(N)
\end{aligned}
$$

with

$$
F_{i}:=8 a\left(1 /\left(2 \kappa_{i}\right)\right)+G_{i} \quad(i=1,2)
$$

and

$$
G_{i}:=8 h\left(1 /\left(2 \kappa_{i}\right)\right) \quad(i=1,2) .
$$

$2^{\circ}$ Upper bounds of $\Upsilon_{3}$ and $\Upsilon_{4}$. We divide the sum $\Upsilon_{3}$ (resp. $\Upsilon_{4}$ ) into subsums according to
(a) $N^{\kappa_{1}} \leq p<N^{1 / 4}$,
(b) $N^{1 / 4} \leq p<N^{1 / 2-s_{9} \kappa_{1}}$,
(c) $N^{1 / 2-s_{j} \kappa_{1}} \leq p<N^{1 / 2-s_{j-1} \kappa_{1}}(9 \geq j \geq 4)$,
(d) $N^{1 / 2-s_{3} \kappa_{1}} \leq p<N^{1 / 3}$

(resp. $N^{\kappa_{1}} \leq p<N^{1 / 4}$ or $N^{1 / 4} \leq p<N^{1 / 2-3 \kappa_{1}}$ ), where $s_{i}$ is defined by (3.10). The contribution of (a) is estimated by Proposition 4.1 and we evaluate (b) (resp. $N^{1 / 4} \leq p<N^{1 / 2-3 \kappa_{1}}$ ) by the classical linear sieve. The remaining subsums are treated by Proposition 4.4. It is worth pointing out that case (b) requires another treatment because $\Psi_{1}\left(s_{10}\right)=0$ (see Table 3 below). Thus we obtain

$$
\Upsilon_{i} \leq\left\{F_{i}+o(1)\right\} \Theta(N) \quad(i=3,4),
$$

where

$$
\begin{aligned}
& F_{3}:=8 \int_{1 /\left(6 \kappa_{1}\right)}^{1 /\left(2 \kappa_{1}\right)-1} \frac{A(t)}{t\left(1-2 \kappa_{1} t\right)} d t-G_{3}, \\
& F_{4}:=8 \int_{3}^{1 /\left(2 \kappa_{1}\right)-1} \frac{A(t)}{t\left(1-2 \kappa_{1} t\right)} d t-G_{4},
\end{aligned}
$$


and

$$
\begin{aligned}
G_{4}:= & 8 \int_{1 /\left(4 \kappa_{1}\right)}^{1 /\left(2 \kappa_{1}\right)-1} \frac{H(t)}{t\left(1-2 \kappa_{1} t\right)} d t, \\
G_{3}:=8 & \int_{1 /\left(4 \kappa_{1}\right)}^{1 /\left(2 \kappa_{1}\right)-1} \frac{H(t)}{t\left(1-2 \kappa_{1} t\right)} d t+8 \int_{1 /\left(6 \kappa_{1}\right)}^{s_{3}} \frac{\Psi_{2}\left(s_{3}\right)}{t(1-2 \kappa t)} d t \\
& +8 \sum_{4 \leq i \leq 5} \int_{s_{i-1}}^{s_{i}} \frac{\Psi_{2}\left(s_{i}\right)}{t(1-2 \kappa t)} d t+8 \sum_{6 \leq i \leq 9} \int_{s_{i-1}}^{s_{i}} \frac{\Psi_{1}\left(s_{i}\right)}{t(1-2 \kappa t)} d t .
\end{aligned}
$$

$3^{\circ}$ Lower bounds of $\Upsilon_{5}$ and $\Upsilon_{6}$. Since $\kappa_{1}+2 \kappa_{2}=0.318 \ldots<1 / 2$, Proposition 4.2 yields

$$
\Upsilon_{5} \geq\left\{F_{5}+o(1)\right\} \Theta(N)
$$

where

$$
\begin{aligned}
F_{5} & :=8 \int_{\kappa_{1}}^{\kappa_{2}} \int_{\left(1 / 2-\kappa_{2}-t\right) / \kappa_{1}}^{(1 / 2-2 t) / \kappa_{1}} \frac{a(u) d t d u}{t u\left(1-2 t-2 \kappa_{1} u\right)}+G_{5}, \\
G_{5} & :=8 \int_{\kappa_{1}}^{\kappa_{2}} \int_{\left(1 / 2-\kappa_{2}-t\right) / \kappa_{1}}^{(1 / 2-2 t) / \kappa_{1}} \frac{h(u) d t d u}{t u\left(1-2 t-2 \kappa_{1} u\right)} .
\end{aligned}
$$

We divide the double sum $\Upsilon_{6}$ into three subsums according to

(a) $N^{\kappa_{1}} \leq p_{1}<N^{\kappa_{2}} \leq p_{2}<N^{1 / 2-2 \kappa_{2}}$,

(b) $N^{\kappa_{1}} \leq p_{1}<N^{3 \kappa_{1} / 2}$ and $N^{1 / 2-2 \kappa_{2}} \leq p_{2}<N^{1 / 2-3 \kappa_{1}}$,

(c) $N^{3 \kappa_{1} / 2} \leq p_{1}<N^{\kappa_{2}}$ and $N^{1 / 2-2 \kappa_{2}} \leq p_{2}<N^{1 / 2-3 \kappa_{1}}$.

The first two subsums can be estimated by Proposition 4.3 and the last one by the classical linear sieve. Thus we obtain

$$
\Upsilon_{6} \geq\left\{F_{6}+o(1)\right\} \Theta(N)
$$

where

$$
\begin{aligned}
& F_{6}:= 8 \int_{\kappa_{1}}^{\kappa_{2}} \int_{\left(3 \kappa_{1}-t\right) / \kappa_{1}}^{\left(1 / 2-\kappa_{2}-t\right) / \kappa_{1}} \frac{a(u) d t d u}{t u\left(1-2 t-2 \kappa_{1} u\right)}+G_{6}, \\
& G_{6}:=8 \int_{\kappa_{1}}^{\kappa_{2}} \int_{\left(2 / 2-\kappa_{2}-t\right) / \kappa_{1}}^{(1 / t) / \kappa_{1}} \frac{h(u) d t d u}{t u\left(1-2 t-2 \kappa_{1} u\right)} \\
&+8 \int_{\kappa_{1}}^{3 \kappa_{1} / 2} \int_{\left(3 \kappa_{1}-t\right) / \kappa_{1}}^{\left(2 \kappa_{2}-t\right) / \kappa_{1}} \frac{h(u) d t d u}{t u\left(1-2 t-2 \kappa_{1} u\right)} .
\end{aligned}
$$


$4^{\mathrm{o}}$ Upper bounds of $\Upsilon_{i}$ for $i=7,8,9,10,11$. Clearly the terms $\Upsilon_{7}, \Upsilon_{8}, \Upsilon_{9}$, $\Upsilon_{10}$ and $\Upsilon_{11}$ here are the terms $\Upsilon_{7}$ (with $\left.\sigma_{1}=1 / 2-3 \kappa_{1}\right), \Upsilon_{9}$ (with $\sigma_{1}=1 / 3$ ), $\Upsilon_{10}$ (with $\left.\sigma_{2}=1 / 2-3 \kappa_{1}\right), \Upsilon_{13}$ and $\Upsilon_{14}$ of (9.4) in [13]. Thus (10.10), (10.11), (10.12) of [13] give us the estimates

$$
\Upsilon_{i} \leq\left\{F_{i}+o(1)\right\} \Theta(N) \quad(i=7,8,9,10,11),
$$

where

$$
\begin{aligned}
& F_{7}:=8 \int_{2}^{2 /\left(1-6 \kappa_{1}\right)-1} \frac{\log (t-1)}{t} d t \\
& F_{8}:=\frac{36}{5} \int_{\kappa_{1}}^{1 / 10} \frac{\log (2-3 t)}{t(1-t)^{2}} d t+8 \int_{1 / 10}^{1 / 3} \frac{\log (2-3 t)}{t(1-t)} d t, \\
& F_{9}:=8 \int_{\kappa_{2}}^{1 / 2-3 \kappa_{1}} \frac{\log \left\{\left(1+6 \kappa_{1}-2 t\right) /\left(1-6 \kappa_{1}\right)\right\}}{t(1-t)} d t, \\
& F_{10}:=\frac{36}{5} \int_{\kappa_{1}}^{1 / 10} \frac{d t_{1}}{t_{1}\left(1-t_{1}\right)} \int_{t_{1}}^{\kappa_{2}} \frac{d t_{2}}{t_{2}^{2}} \int_{t_{2}}^{\kappa_{2}} \frac{d t_{3}}{t_{3}} \int_{t_{3}}^{\kappa_{2}} \omega\left(\frac{1-t_{1}-t_{2}-t_{3}-t_{4}}{t_{2}}\right) \frac{d t_{4}}{t_{4}} \\
& +8 \int_{1 / 10}^{\kappa_{2}} \frac{d t_{1}}{t_{1}} \int_{t_{1}}^{\kappa_{2}} \frac{d t_{2}}{t_{2}^{2}} \int_{t_{2}}^{\kappa_{2}} \frac{d t_{3}}{t_{3}} \int_{t_{3}}^{\kappa_{2}} \omega\left(\frac{1-t_{1}-t_{2}-t_{3}-t_{4}}{t_{2}}\right) \frac{d t_{4}}{t_{4}}, \\
& F_{11}:=\frac{36}{5} \int_{\kappa_{1}}^{1 / 10} \frac{d t_{1}}{t_{1}\left(1-t_{1}\right)} \int_{t_{1}}^{\kappa_{2}} \frac{d t_{2}}{t_{2}^{2}} \int_{t_{2}}^{\kappa_{2}} \frac{d t_{3}}{t_{3}} \\
& \times \int_{\kappa_{2}}^{1 / 2-2 \kappa_{1}-t_{3}} \omega\left(\frac{1-t_{1}-t_{2}-t_{3}-t_{4}}{t_{2}}\right) \frac{d t_{4}}{t_{4}} \\
& +8 \int_{1 / 10}^{\kappa_{2}} \frac{d t_{1}}{t_{1}} \int_{t_{1}}^{\kappa_{2}} \frac{d t_{2}}{t_{2}^{2}} \int_{t_{2}}^{\kappa_{2}} \frac{d t_{3}}{t_{3}} \int_{\kappa_{2}}^{1 / 2-2 \kappa_{1}-t_{3}} \omega\left(\frac{1-t_{1}-t_{2}-t_{3}-t_{4}}{t_{2}}\right) \frac{d t_{4}}{t_{4}},
\end{aligned}
$$

and $\omega(t)$ is the Buchstab function (see Lemma 2.10 of [13]).

Inserting (5.2)-(5.6) into (2.2), we get

$$
D_{1,2}(N) \geq\left\{F\left(\kappa_{1}, \kappa_{2}\right)+o(1)\right\} \Theta(N),
$$

where

$$
\begin{aligned}
& F\left(\kappa_{1}, \kappa_{2}\right) \\
& \quad:=\frac{1}{4}\left(3 F_{1}+F_{2}-F_{3}-F_{4}+F_{5}+F_{6}-2 F_{7}-F_{8}-F_{9}-F_{10}-F_{11}\right) .
\end{aligned}
$$


$5^{\mathrm{o}}$ Numerical computation. From (3.2) and (3.3), we deduce easily that

$$
a(s)= \begin{cases}0 & (0<s \leq 2), \\ \log (s-1) & (2<s \leq 4), \\ \log (s-1)+\int_{3}^{s-1} \frac{d t}{t} \int_{2}^{t-1} \frac{\log (u-1)}{u} d u & (4<s \leq 6), \\ \log (s-1)+\int_{3}^{s-1} \frac{d t}{t} \int_{2}^{t-1} \frac{\log (u-1)}{u} d u & \\ \quad+\int_{5}^{s-1} \frac{d t}{t} \int_{4}^{t-1} \frac{d u}{u} \int_{3}^{u-1} \frac{d v}{v} \int_{2}^{v-1} \frac{\log (w-1)}{w} d w & (6<s \leq 8),\end{cases}
$$

and

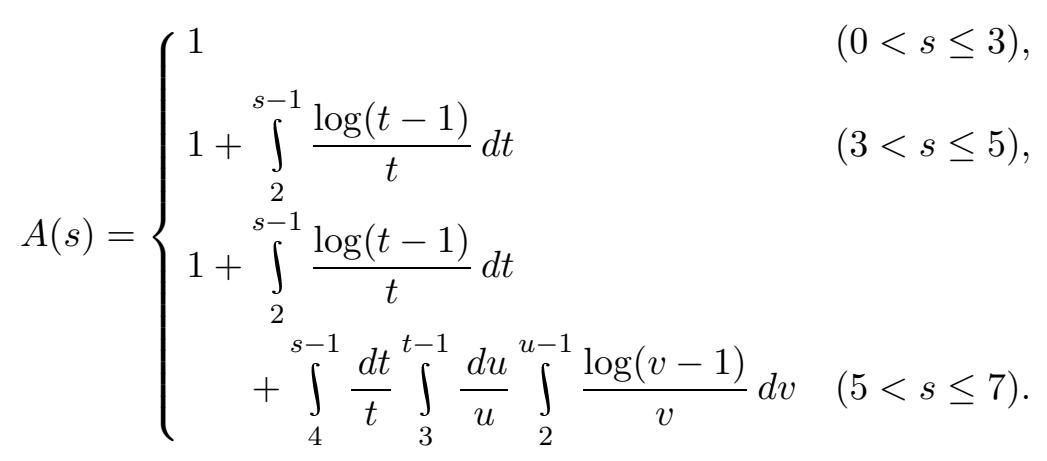

By using (3.8), we have

$$
G_{2} \geq 8\left(h\left(s_{22}\right)+\int_{s_{22}-1}^{1 /\left(2 \kappa_{2}\right)-1} \frac{H(t)}{t} d t\right) \geq 0.005283 .
$$

In order to estimate $G_{4}$, we use Table 1 and the decreasing of $H(s)$ to obtain

$$
G_{4}=8 \int_{1 /\left(4 \kappa_{1}\right)}^{1 /\left(2 \kappa_{1}\right)-1} \frac{H(t)}{t\left(1-2 \kappa_{1} t\right)} d t \geq 8 \sum_{14 \leq i \leq 29} g_{4}^{i} H\left(s_{i}\right) \geq 0.008860
$$

with

$$
\begin{aligned}
g_{4}^{14} & :=\log \left(\frac{2 \kappa_{1} s_{14}}{1-2 \kappa_{1} s_{14}}\right) \\
g_{4}^{i} & :=\log \left(\frac{s_{i}\left(1-2 \kappa_{1} s_{i-1}\right)}{s_{i-1}\left(1-2 \kappa_{1} s_{i}\right)}\right) \quad(15 \leq i \leq 29) .
\end{aligned}
$$

With a simpler calculation, we get

$$
G_{3}=G_{4}+8 \sum_{3 \leq i \leq 5} g_{3}^{i} \Psi_{2}\left(s_{i}\right)+8 \sum_{6 \leq i \leq 9} g_{3}^{i} \Psi_{1}\left(s_{i}\right) \geq 0.039890
$$


with

$$
\begin{aligned}
g_{3}^{3} & :=\log \left(\frac{4 \kappa_{1} s_{3}}{1-2 \kappa_{1} s_{3}}\right), \\
g_{3}^{i} & :=\log \left(\frac{s_{i}\left(1-2 \kappa_{1} s_{i-1}\right)}{s_{i-1}\left(1-2 \kappa_{1} s_{i}\right)}\right) \quad(4 \leq i \leq 9) .
\end{aligned}
$$

Here we have used Table 1 of [13] on the lower bounds for $\Psi_{2}\left(s_{i}\right)(3 \leq i \leq 5)$ and $\Psi_{1}\left(s_{i}\right)(6 \leq i \leq 9)$ :

Table 3. Lower bounds for $\Psi_{1}\left(s_{i}\right)$ and $\Psi_{2}\left(s_{i}\right)$

\begin{tabular}{cccccccc}
\hline$i$ & $s_{i}$ & $s_{i}^{\prime}$ & $\kappa_{1, i}$ & $\kappa_{2, i}$ & $\kappa_{3, i}$ & $\Psi_{1}\left(s_{i}\right)$ & $\Psi_{2}\left(s_{i}\right)$ \\
\hline 3 & 2.3 & 4.50 & 3.54 & 2.88 & 2.43 & & 0.015247971 \\
4 & 2.4 & 4.46 & 3.57 & 2.87 & 2.40 & & 0.013898757 \\
5 & 2.5 & 4.12 & 3.56 & 2.91 & 2.50 & & 0.011776059 \\
6 & 2.6 & 3.58 & & & & 0.009405211 & \\
7 & 2.7 & 3.47 & & & & 0.006558950 & \\
8 & 2.8 & 3.34 & & & & 0.003536751 & \\
9 & 2.9 & 3.19 & & & & 0.001056651 & \\
10 & 3.0 & 3.00 & & & & 0 & \\
\hline
\end{tabular}

Similarly

$$
\begin{aligned}
G_{5} & =8 \int_{\kappa_{1}}^{\kappa_{2}} \int_{\left(1 / 2-\kappa_{2}-t\right) / \kappa_{1}}^{(1 / 2-2 t) / \kappa_{1}} \frac{h(u) d t d u}{t u\left(1-2 t-2 \kappa_{1} u\right)} \\
& =8 \int_{\left(1 / 2-2 \kappa_{2}\right) / \kappa_{1}}^{\left(1 / 2-\kappa_{1}-\kappa_{2}\right) / \kappa_{1}} h(u) \log \left(\frac{2 \kappa_{2}}{1-2 \kappa_{2}-2 \kappa_{1} u}\right) \frac{d u}{u\left(1-2 \kappa_{1} u\right)} \\
& +8 \int_{\left(1 / 2-2 \kappa_{1}\right) / \kappa_{1}}^{\left(1 / 2-\kappa_{1}-\kappa_{2}\right) / \kappa_{1}} h(u) \log \left(\frac{1-2 \kappa_{1}-2 \kappa_{1} u}{2 \kappa_{1}}\right) \frac{d u}{u\left(1-2 \kappa_{1} u\right)} \\
\geq & \sum_{15 \leq i \leq 27}^{i} g_{5}^{i} h\left(s_{i}\right) \geq 0.001359
\end{aligned}
$$

with

$$
\begin{aligned}
g_{5}^{15} & :=\int_{\left(1 / 2-2 \kappa_{2}\right) / \kappa_{1}}^{s_{15}} \log \left(\frac{2 \kappa_{2}}{1-2 \kappa_{2}-2 \kappa_{1} u}\right) \frac{d u}{u\left(1-2 \kappa_{1} u\right)}, \\
g_{5}^{i} & :=\int_{s_{i-1}}^{s_{i}} \log \left(\frac{2 \kappa_{2}}{1-2 \kappa_{2}-2 \kappa_{1} u}\right) \frac{d u}{u\left(1-2 \kappa_{1} u\right)} \quad(16 \leq i \leq 20),
\end{aligned}
$$




$$
\begin{aligned}
g_{5}^{21}:= & \int_{s_{20}}^{\left(1 / 2-\kappa_{1}-\kappa_{2}\right) / \kappa_{1}} \log \left(\frac{2 \kappa_{2}}{1-2 \kappa_{2}-2 \kappa_{1} u}\right) \frac{d u}{u\left(1-2 \kappa_{1} u\right)} \\
& +\int_{\left(1 / 2-\kappa_{1}-\kappa_{2}\right) / \kappa_{1}}^{s_{21}} \frac{\log \left(1 /\left(2 \kappa_{1}\right)-1-u\right)}{u\left(1-2 \kappa_{1} u\right)} d u, \\
g_{5}^{i}:= & \int_{s_{i-1}}^{s_{i}} \frac{\log \left(1 /\left(2 \kappa_{1}\right)-1-u\right)}{u\left(1-2 \kappa_{1} u\right)} d u \quad(22 \leq i \leq 26), \\
g_{5}^{27}:= & \int_{s_{26}} \frac{\log \left(1 /\left(2 \kappa_{1}\right)-1-u\right)}{u\left(1-2 \kappa_{1} u\right)} d u
\end{aligned}
$$

and

$$
\begin{aligned}
G_{6}= & 8 \int_{\kappa_{1}}^{\kappa_{2}} \int_{\left(2 \kappa_{2}-t\right) / \kappa_{1}}^{\left(1 / 2-\kappa_{2}-t\right) / \kappa_{1}} \frac{h(u) d t d u}{t u\left(1-2 t-2 \kappa_{1} u\right)} \\
& +8 \int_{\kappa_{1}}^{3 \kappa_{1} / 2\left(2 \kappa_{2}-t\right) / \kappa_{1}} \int_{\left(3 \kappa_{1}-t\right) / \kappa_{1}}^{t u\left(1-2 t-2 \kappa_{1} u\right)} \frac{h(u) d t d u}{t\left(1 / 2-2 \kappa_{2}\right) / \kappa_{1}} \log \left(\frac{\kappa_{2}\left(1-2 \kappa_{1}-2 \kappa_{1} u\right)}{\kappa_{1}\left(1-2 \kappa_{2}-2 \kappa_{1} u\right)}\right) \frac{h(u) d u}{u\left(1-2 \kappa_{1} u\right)} \\
\geq & \quad \int_{2}^{\left(1 / 2-\kappa_{1}-\kappa_{2}\right) / \kappa_{1}} \log \left(\frac{\left(1-2 \kappa_{1}-2 \kappa_{1} u\right)\left(1-2 \kappa_{2}-2 \kappa_{1} u\right)}{4 \kappa_{1} \kappa_{2}}\right) \frac{h(u) d u}{u\left(1-2 \kappa_{1} u\right)} \\
& +8 \int_{1 \leq i \leq 21}^{\left(1 / 2-2 \kappa_{2}\right) / \kappa_{1}}
\end{aligned}
$$

with

$$
\begin{aligned}
& g_{6}^{i}:= \int_{s_{i-1}}^{s_{i}} \log \left(\frac{\kappa_{2}\left(1-2 \kappa_{1}-2 \kappa_{1} u\right)}{\kappa_{1}\left(1-2 \kappa_{2}-2 \kappa_{1} u\right)}\right) \frac{d u}{u\left(1-2 \kappa_{1} u\right)} \quad(1 \leq i \leq 14), \\
& g_{6}^{15}:= \int_{s_{14}}^{\left(1 / 2-2 \kappa_{2}\right) / \kappa_{1}} \log \left(\frac{\kappa_{2}\left(1-2 \kappa_{1}-2 \kappa_{1} u\right)}{\kappa_{1}\left(1-2 \kappa_{2}-2 \kappa_{1} u\right)}\right) \frac{d u}{u\left(1-2 \kappa_{1} u\right)} \\
&+\int_{g_{6}:}^{s_{15}} \log \left(\frac{\left(1-2 \kappa_{1}-2 \kappa_{1} u\right)\left(1-2 \kappa_{2}-2 \kappa_{1} u\right)}{4 \kappa_{1} \kappa_{2}}\right) \frac{d u}{u\left(1-2 \kappa_{1} u\right)}, \\
& g_{s_{i-1}}^{i} \log \left(\frac{\left(1-2 \kappa_{1}-2 \kappa_{1} u\right)\left(1-2 \kappa_{2}-2 \kappa_{1} u\right)}{4 \kappa_{1} \kappa_{2}}\right) \frac{d u}{u\left(1-2 \kappa_{1} u\right)}
\end{aligned}
$$$$
(16 \leq i \leq 20),
$$ 


$$
g_{6}^{21}:=\int_{s_{20}}^{\left(1 / 2-\kappa_{1}-\kappa_{2}\right) / \kappa_{1}} \log \left(\frac{\left(1-2 \kappa_{1}-2 \kappa_{1} u\right)\left(1-2 \kappa_{2}-2 \kappa_{1} u\right)}{4 \kappa_{1} \kappa_{2}}\right) \frac{d u}{u\left(1-2 \kappa_{1} u\right)} .
$$

To simplify the computation of $F_{10}$ and $F_{11}$, we make use of the fact that $\omega(t) \leq 0.561522$ for $t \geq 3.4$.

Finally, a numerical computation yields

$$
\begin{aligned}
F\left(\kappa_{1}, \kappa_{2}\right) \geq & \frac{1}{4}\{3 \cdot 14.900897+(9.103015+0.005283) \\
& -(23.652925-0.039890)-(19.643510-0.008860) \\
& +(1.654808+0.001359)+(3.819092+0.060469) \\
& -2 \cdot 0.585179-5.279581-5.372410-0.104305-0.543858\} \\
> & 0.899 .
\end{aligned}
$$

This completes the proof of the Theorem.

\section{References}

[1] E. Bombieri and H. Davenport, Small differences between prime numbers, Proc. Roy. Soc. Ser. A 293 (1966), 1-18.

[2] Y. C. Cai, On Chen's theorem (II), J. Number Theory, to appear.

[3] —, Chen's theorem with small primes, Acta Math. Sinica (Chin. Ser.) 48 (2005), 593-598 (in Chinese).

[4] Y. C. Cai and M. G. Lu, On Chen's theorem, in: Analytic Number Theory (Beijing/Kyoto, 1999), Dev. Math. 6, Kluwer, Dordrecht, 2002, 99-119.

[5] J. R. Chen, On the representation of a large even integer as the sum of a prime and the product of at most two primes, Sci. Sinica 16 (1973), 157-176.

[6] - On the representation of a large even integer as the sum of a prime and the product of at most two primes (II), ibid. 21 (1978), 421-430.

[7] -, Further improvement on the constant in the proposition '1+2': On the representation of a large even integer as the sum of a prime and the product of at most two primes (II), ibid. 21 (1978), 477-494 (in Chinese).

[8] - On the Goldbach's problem and the sieve methods, ibid. 21 (1978), 701-739.

[9] H. Halberstam and H.-E. Richert, Sieve Methods, Academic Press, London, 1974.

[10] G. H. Hardy and J. E. Littlewood, Some problems of 'partitio numerorum' III: On the expression of a number as a sum of primes, Acta Math. 44 (1923), 1-70.

[11] C. D. Pan and C. B. Pan, Goldbach Conjecture, Science Press, Beijing, 1992.

[12] J. Wu, Sur la suite des nombres premiers jumeaux, Acta Arith. 55 (1990), 365-394.

[13] —, Chen's double sieve, Goldbach's conjecture and the twin prime problem, ibid. 114 (2004), 215-273.

Institut Élie Cartan Nancy (IECN)

Nancy-Université, CNRS, INRIA

Boulevard des Aiguillettes, B.P. 239

54506 Vandœuvre-lès-Nancy, France

E-mail: wujie@iecn.u-nancy.fr
School of Mathematical Sciences

Shandong Normal University Jinan, Shandong, 250014, P.R. China 